\title{
Preparation and Characterization of Soluble Eggshell Membrane Protein/PLGA Electrospun Nanofibers for Guided Tissue Regeneration Membrane
}

\author{
Jun Jia, ${ }^{1}$ Geng Liu,, ${ }^{1}$ hao-Xia Guo, ${ }^{2}$ Jian Yu, ${ }^{2}$ and Yuanyuan Duan ${ }^{1}$ \\ ${ }^{1}$ Department of Prosthodontics, School of Stomatology, Fourth Military Medical University, Xi'an 710032, China \\ ${ }^{2}$ Institute of Polymer Science and Engineering, School of Materials Science and Engineering, Tsinghua University, \\ Beijing 100084, China
}

Correspondence should be addressed to Yuanyuan Duan, yyduan@gmail.com

Received 4 October 2011; Revised 30 November 2011; Accepted 7 December 2011

Academic Editor: Suprakas Sinha Ray

Copyright ( 2012 Jun Jia et al. This is an open access article distributed under the Creative Commons Attribution License, which permits unrestricted use, distribution, and reproduction in any medium, provided the original work is properly cited.

\begin{abstract}
Guided tissue regeneration (GTR) is a widely used method in periodontal therapy, which involves the placement of a barrier membrane to exclude migration of epithelium and ensure repopulation of periodontal ligament cells. The objective of this study is to prepare and evaluate a new type of soluble eggshell membrane protein (SEP)/poly (lactic-co-glycolic acid) (PLGA) nanofibers using electrospinning method for GTR membrane application. SEP/PLGA nanofibers were successfully prepared with various blending ratios. The morphology, chemical composition, surface wettability, and mechanical properties of the nanofibers were characterized using scanning electron microscopy (SEM), contact angle measurement, Fourier transform-infrared spectroscopy (FTIR), and a universal testing machine. L-929 fibroblast cells were used to evaluate the biocompatibility of SEP/PLGA nanofibers and investigate the interaction between cells and nanofibers. Results showed that the SEP/PLGA electrospun membrane was composed of uniform, bead-free nanofibers, which formed an interconnected porous network structure. Mechanical property of SEP has been greatly improved by the addition of PLGA. The biological study results showed that SEP/PLGA nanofibers could enhance cell attachment, spreading, and proliferation. The study indicated the potential of SEP/PLGA nanofibers for GTR application and provided a basis for future optimization.
\end{abstract}

\section{Introduction}

Periodontal disease that involves the deterioration of toothsupporting structures is one of the most prevalent oral diseases all over the world. It affects 5-15 percent of human population and is the primary cause of tooth loss among adults [1]. To regenerate the damaged periodontal tissue and supporting bone, a surgical approach is widely performed by placing a space-maintaining barrier membrane between the root surface and the gingival flap. This technique, known as guided tissue regeneration (GTR), is based on the exclusion of gingival epithelial cell from the damaged area by using a barrier membrane, hence allowing the selective repopulation of tissues derived from the periodontal ligament and alveolar bone. There are a large variety of nonresorbable and resorbable commercially available GTR membranes. Nonresorbable GTR membranes, such as expanded polytetrafluoroethylene (e-PTFE), provide favorable mechanical property and structural integrity but require extra surgical procedure for the removal [2]. Therefore, bioresorbable membranes have been increasingly used, which mainly include natural biopolymers like collagen and synthetic polymers such as poly-lactic acid (PLA). However, nature-derived GTR membranes usually have satisfactory biocompatibility but relatively inadequate space-maintaining ability, while the synthetic polymer membranes show poor biocompatibility and possible inflammatory response due to their acid degradation products [3]. Hence, new approaches are needed to develop a satisfactory barrier membrane which has favorable biocompatibility as well as adequate mechanical strength and space-maintaining ability, in order to fulfill clinically predictable periodontal tissue regeneration. 
Eggshell membrane (ESM) is a bilayered barrier membrane between egg white and eggshell. It has an abundant, cost-effective resource from the waste materials of food industry [4]. ESM mainly consists of proteins such as collagen (types I, V, and X), osteopontin, and sialoprotein. Both of the outer and inner membranes are composed of interwoven protein fibers, while the inner membrane is comparably thicker and more compact [5]. ESM permits gaseous exchange, protects the chicken embryo just as the human amniotic membrane does to human fetus and specifically plays a key role in the biomineralization of eggshell, which only takes less than $24 \mathrm{~h}$ and is the fastest biomineralization process we have ever known $[6,7]$. In Chinese traditional medicine, ESM was formally named as "phoenix cloth" and frequently used for treating the chronic ulcers and bone fractures since many centuries. In recent decades, ESM has been proved to have great biocompatibility and reported to give satisfactory results as a biological dressing for burns or skin graft donor sites [8-10]. It is quite interesting to compare the features of natural ESM to the demands of GTR membrane, since both of them serve as protective physical barrier as well as have the beneficial effects on tissue regeneration. Inspired by these features, Dupoirieux tried to use natural ESM directly as the GTR membrane, but finally failed due to its poor space-maintaining ability [11]. In order to facilitate the biomedical applications of ESM, Yi successfully prepared soluble eggshell membrane protein (SEP) from natural ESM by using aqueous 3 -mercaptopropionic acid and acetic acid $[12,13]$. The acquired SEP is soluble in common nontoxic solvents which allow more ease for further modification and processing. It has been proved that the biocompatibility of this SEP product is comparable to collagen type I, however, still quite weak and brittle in mechanical strength. After that, great efforts have been made to further improve the properties of SEP such as blending with synthetic polymers, surface physical entrapment, and plasma immobilization $[14,15]$. But none of them could mimic the natural structure of ESM, and there was no report of SEP for periodontal GTR membrane application either.

Electrospinning method is a fast and simple fabrication process which can generate nanosized polymer fibers by applying a strong electric field to a liquid polymer droplet. It has received great attention in recent years and widely used to produce nanofibrous scaffolds for wound dressing, medical implant materials, drug delivery, and tissue engineering applications [16-18]. The electrospun fibers have nanoscale diameters, very high surface to volume ratio and a fully interconnected porous network structure which can utmostly mimic the features of natural extracelluar matrix (ECM), which is also very similar to the structure of ESM $[19,20]$. In this study, we propose that a new type of nanofibrous membrane can be developed by the combination of SEP preparation and electrospinning technique. This new membrane will mimic the chemical composition and porous structure of the natural ESM. It can physically maintain the space for the tissue regeneration, exclude the invasion of epithelial tissue as well as enhance the attachment, proliferation, and differentiation of periodontal ligament cells.
However, it was observed in pilot study that the pure SEP product has a relatively poor electrospinnability probably due to the low molecular weight and inadequate solubility in solvents. Pure SEP fibers have been formed occasionally in the pilot study, but the fibers did not have acceptable morphology and were accompanied by a lot of dispersing liquid droplets. The pure SEP fibers were also very fragile and not ready for any further use. Apparently, the electrospinnability of SEP needs to be improved before it can be used as candidate for GTR membrane. Blendelectrospinning method is used in the study to improve the electrospinnability and mechanical properties of SEP. Yi et al. [21] has prepared the poly (ethylene oxide) (PEO)/SEP nanofibers by electrospinning successfully, while the nanofibers need to be treated by cross-linking agent due to the quick and complete dissolution in the water. The similar phenomenon was observed in another study, and the fibers were treated by catechin to improve the water insolubility [22]. While in these studies, the toxicity of crosslinking agents still remains a concern. Poly lactic-co-glycolic acid (PLGA) has a long history of clinical use and has been widely used in medical and pharmaceutical applications due to its good biocompatibility and mechanical property. The electrospinning of PLGA has been accomplished in 2002 [23], and it has been successfully blended with a variety of natural biopolymers such as collagen, chitosan, gelatin, and silk fibroin for better electrospinnability and mechanical strength [24-27]. Hence, in this study, PLGA is used to blend with SEP for the preparation of electrospun nanofibers with various process parameters such as solution concentrations and blending ratios. The chemical, surface, mechanical, and biological properties of the fabricated fibrous membrane will be characterized and evaluated for the further GTR applications.

\section{Materials and Methods}

2.1. Materials. Raw ESM was obtained manually from commercial hen eggs and powdered by liquid nitrogen grinding method with a mortar and pestle. SEP was prepared by dissolving raw ESM powder in aqueous 3-mercaptopropionic acid and acetic acid followed by neutralizing to $\mathrm{pH} 5$ which has been detailed described in our previous study $[12,13]$. PLGA $(\mathrm{Mn}=100,000, \mathrm{LA}: \mathrm{GA}=80: 20)$ and $1,1,1,3,3,3$ hexafluoro-2-propanol (HFIP) were purchased from Sigma Aldrich (St. Louis, USA) and used as received according to the instructions of manufacturer.

2.2. Electrospinning. Spinning solutions were prepared by dissolving PLGA and SEP in HFIP with concentrations of $10 \%(\mathrm{w} / \mathrm{v})$ at three different blending ratios $(\mathrm{SEP} / \mathrm{PLGA}=$ $90: 10,70: 30$, and 50:50). All mixtures were vigorously stirred at room temperature for $12 \mathrm{~h}$. An electrospinning apparatus was designed and built, composing of a syringe and stainless needle, a ground electrode, a copper plate covered by aluminum foil as a collector, and an adjustable high voltage supply (Model BGG40/2, BMEI Co., Beijing, China). The prepared solution was filled into the $5 \mathrm{~mL}$ 
syringe, and anode is attached to the external surface of the metal needle. When the high voltage was applied across the syringe and the grounded collector, the solution would be ejected from the tip of the needle to produce nanofibers and deposit on the grounded collector as fibrous membranes. After the pilot study, the electrospinning was operated at a voltage of $17 \mathrm{kV}$ and a working distance of $14 \mathrm{~cm}$ (the distance between the needle tip and the collector). The flow rate of solution was $0.6 \mathrm{~mL} / \mathrm{h}$. The as-spun mats were dried under vacuum for $24 \mathrm{~h}$ to remove residual solvent and then used for further studies.

2.3. Morphology, Contact Angle Measurements, and FTIR Spectroscopy. Electrospun fibers were gold-sputtered and then observed using SEM (JSM-6390A, JEOL, Japan). The average diameters were determined by analyzing SEM images with Image J 1.36b software (National Institute of Health, USA). Surface wettability of electrospun membrane was evaluated by measuring glycerol contact angles using a sessile drop method on a Drop-Shape Analysis System DSA 10 (Kruss, Hamburg, Germany) at room temperature. The measurements were repeated at five different sites on each sample. Fourier transforms-infrared spectrometer (AVATAR 360, Nicolet, USA) was used to obtain the spectra of the PLGA fibers, SEP/PLGA blended fibers, and raw SEP product, respectively.

2.4. Mechanical Tests. Mechanical properties of the nanofibrous membranes are measured with a tabletop tester (EZ Test, Shimadzu, Japan) with a crosshead speed of $5 \mathrm{~mm} / \mathrm{min}$. The samples were carefully prepared and cut into the rectangular shape with the dimension of $5 \mathrm{~mm}$ in width and $50 \mathrm{~mm}$ in length. Both ends of the samples were strengthened by the double-side tape and adhesive plaster to prevent the samples from breaking from the ends. The tensile strength and elongation at break were determined by the software from the manufacturer of the testing machine.

2.5. Cell Morphology and Proliferation Tests. The mousederived L-929 fibroblasts were cultured in the Dulbecco's modified Eagle medium (DMEM) (Sigma, USA) containing $10 \%$ fetal bovine serum (FBS) (Hyclone, UK) in a humidified atmosphere of $95 \%$ air and $5 \% \mathrm{CO}_{2}$ at $37^{\circ} \mathrm{C}$. Cells in passage 3 were used for the following experiments. The blending SEP/PLGA nanofibrous membranes were sterilized by ultraviolet light for $24 \mathrm{~h}$, placed in a 12 -well culture plate, and preimmersed in DMEM for $24 \mathrm{~h}$. The cells were seeded on the membrane samples with a density of $1 \times$ $10^{5} \mathrm{cells} / \mathrm{cm}^{2}$. After $1,3,5$ and 7 days of culture, samples were rinsed twice with phosphate-buffered saline (PBS) and subsequently fixed in $3 \%$ glutaraldehyde for $2 \mathrm{~h}$ at $4^{\circ} \mathrm{C}$. After being washed gently with PBS to remove the nonadherent cells, the samples were sequentially dehydrated in graded ethanol solutions $(50,70,80,90$, and $100 \%)$ for $10 \mathrm{~min}$ at each time and then underwent carbon dioxide critical point drying. The specimens were sputter coated with gold and observed with SEM (JSM-6390A, JEOL, Japan) to investigate the morphological details of cells and their interaction with the nanofibers. A 2-(4,5-dimethylthiazolyl)3,5-diphenyltetrazolium bromide (MTT) assay was also used to examine the cell viability [28]. After each period of culturing time, MTT solution ( $5 \mathrm{mg} / \mathrm{mL}, 200 \mathrm{uL}$, Sigma) was added to each well. After $4 \mathrm{~h}$ incubation at $37^{\circ} \mathrm{C}$, methylsulfinylmethane was added to completely dissolve the red-colored formazan crystals, and the absorbance was measured by a microplate spectrophotometer (Molecular Devices, Sunnyvale, CA, USA) at $490 \mathrm{~nm}$.

\section{Results and Discussion}

3.1. Morphologies of Nanofibers. Electrospinning was stable and successful with all three blending ratios at the concentration of $10 \%(\mathrm{w} / \mathrm{v})$. Figure 1 shows the morphology of pristine PLGA fibers and SEP/PLGA fibers with different blending ratios examined by SEM. It showed that uniform and beadfree ultrafine fibers were achieved for three blending ratios, and the fibers had a three-dimensional, porous network structure. As described before, electrospinning of pure SEP was not successful with varying electrospinning parameters such as solvents and voltages in the pilot study. The addition of PLGA increased the solution viscosity, formed a more stable liquid jet, and greatly improved the electrospinnability of SEP. The uniform, bead-free, and nanofibrous morphology of SEP/PLGA blending nanofibers is also thought to mimic the natural structure of ECM and facilitate an optimal environment for cells to grow and proliferate [29]. The nanofibers with higher SEP ratio than 50\% had inferior and nonuniform morphology and even smaller average fiber diameters, which not only failed to provide ECMlike environment but also compromised the mechanical properties of the nanofibrous mats. Therefore, they were not included for the further examinations in the study.

The average fiber diameters are listed in Table 1. There was no significant difference between the fiber diameters of pristine PLGA group and 90:10 blending group $(P>$ $0.05)$. However, the fiber diameters of the other two blending groups $(70: 30$ and $50: 50)$ were significantly smaller than those of pristine PLGA group and $90: 10$ group $(P<0.05)$. There was significant difference among the fiber diameters of all three blending groups $(P<0.05)$. The results indicated that the average fiber diameters decreased with the decreasing content of PLGA and increasing content of SEP, which may be attributed to the increase of electrical conductivity induced by the amino and carboxyl groups in SEP. The other possible reason for the smaller fiber diameter is thought to be the decrease in solution viscosity due to the increasing content of SEP. This is agreeable with the previous study that there is an allometrical relationship between the diameter of the fibers and the solution viscosity for electrospinning due to the entanglement of macromolecules in resulted solution [30]. It was proposed that with the increase of concentration, more molecules are entangled which lead to an allometrical law relationship.

3.2. Contact Angle Measurements. The results of glycerol contact angle measurements are listed in Table 1. With 


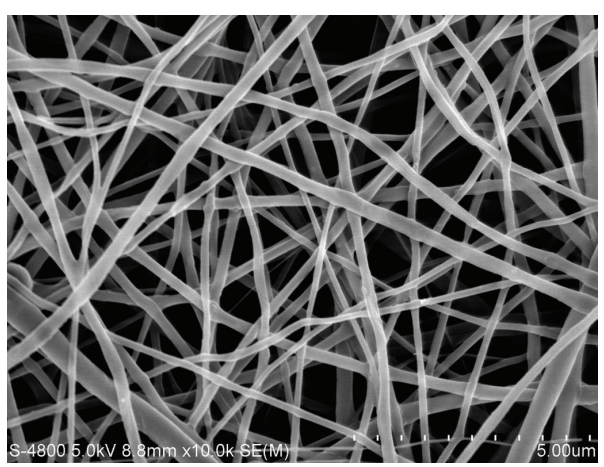

(a)

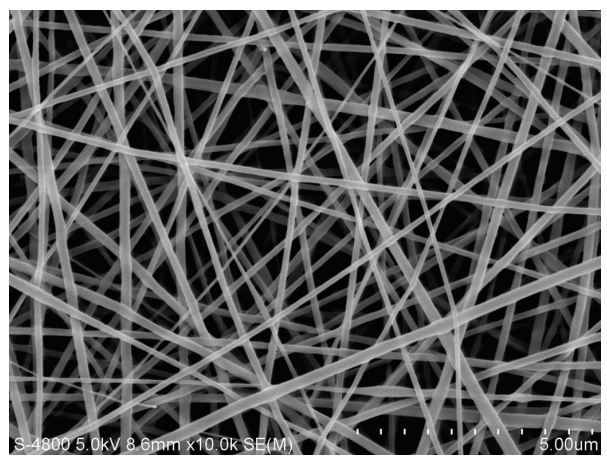

(c)

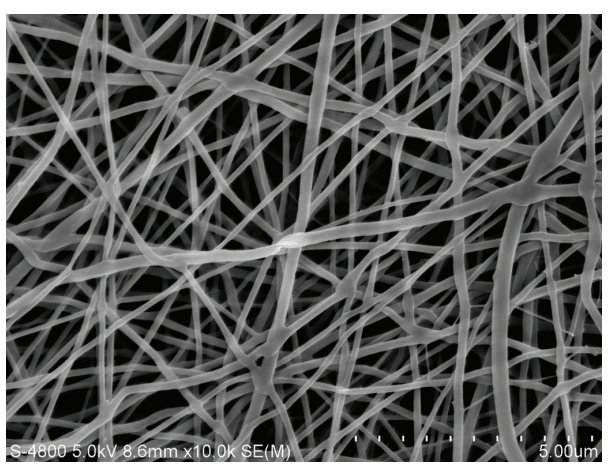

(b)

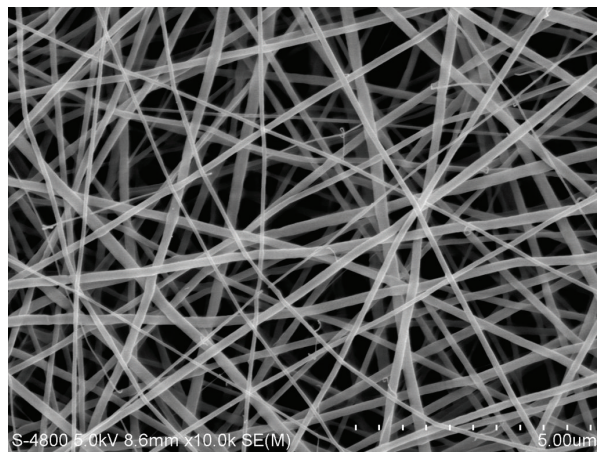

(d)

FIGURE 1: SEM photographs of electrospun fibers with various blending ratios. (a) pristine PLGA; (b) SEP/PLGA = 90 : 10; (c) SEP/PLGA = $70: 30$; (d) SEP/PLGA = 50:50.

TABLE 1: Average fiber diameters and contact angles for PLGA and SEP/PLGA fibers.

\begin{tabular}{lcc}
\hline Group & Diameter $(\mathrm{nm})$ & Contact angle $\left({ }^{\circ} \mathrm{C}\right)$ \\
\hline PLGA & $370.93 \pm 91.41$ & $106.6 \pm 3.01$ \\
SEP/PLGA $=90: 10$ & $335.39 \pm 87.39$ & $103.7 \pm 2.20$ \\
SEP/PLGA $=70: 30$ & $266.06 \pm 76.67$ & $102.6 \pm 1.41$ \\
SEP/PLGA $=50: 50$ & $231.62 \pm 54.88$ & $100.2 \pm 2.57$ \\
\hline
\end{tabular}

the addition of SEP, the contact angle values decreased significantly. There were significant differences between pristine PLGA group and all the blending groups $(P<0.05)$. There was no significant difference among three blending groups $(P>0.05)$. Glycerol was used for contact angle measurements in the study due to the rapid water absorption on the SEP/PLGA fibers. The results showed that the addition of SEP improved the surface wettability of electrospun fibers significantly. It has been known that a hydrophilic surface could enhance the initial attachment, migration, and proliferation of cells via different cellular signaling events $[31,32]$. The surface hydrophilicity due to the presence of SEP could promisingly enhance the cellular behaviors in the further biomedical applications. Besides, contact angle value from the group with the blending ratio of $90: 10$ was significantly smaller than the pristine PLGA group but was not significantly different from the other two groups with higher content of SEP. This indicated that SEP improved the hydrophility of the electrospun nanofibers quite effectively and relatively higher PLGA content in the nanofibers would not compromise the wettability of the material.

3.3. Mechanical Tests. Results of the axial tensile testing are listed in Table 2. There was no significant difference between pristine PLGA group and SEP/PLGA 90:10 group $(P>$ $0.05)$. However, there were significant differences between pristine PLGA group and the other two blending groups $(P<0.05)$. There were also significant differences among all three blending groups $(P<0.05)$. The pristine PLGA group had highest percentage elongation at break compared to the blending groups $(P<0.05)$. The results showed that the pristine PLGA group and 90:10 blending group have best mechanical performance and are more ductile than the other blending groups. With the increasing content of SEP, the tensile strength and elongation at break was decreased due to the inherent weakness of SEP and possibly the phase separation between the two materials. However, in view of the future GTR application, the mechanical strength data of these two groups are still within the satisfactory limits and comparable to the tensile strength data of commercial available GTR membranes in the previous papers $[33,34]$. Li et al. reported that the maximum failure load increases with the increase of thickness for electrospun polymer fibers [35]. The mechanical behaviour of electrospun fibers has also been successfully improved by applying cross-linking agents [21]. In the future in vivo applications, if higher strength 
TABLE 2: Tensile properties of for PLGA and SEP/PLGA fibers.

\begin{tabular}{lcc}
\hline & $\begin{array}{c}\text { Tensile strength } \\
(\mathrm{MPa})\end{array}$ & $\begin{array}{c}\text { Elongation at } \\
\text { break }(\%)\end{array}$ \\
\hline PLGA & $4.64 \pm 0.09$ & $47.06 \pm 3.59$ \\
SEP/PLGA $=90: 10$ & $4.21 \pm 0.28$ & $41.75 \pm 2.82$ \\
SEP/PLGA $=70: 30$ & $2.08 \pm 0.51$ & $22.45 \pm 2.48$ \\
SEP/PLGA $=50: 50$ & $1.16 \pm 0.14$ & $15.91 \pm 1.81$ \\
\hline
\end{tabular}

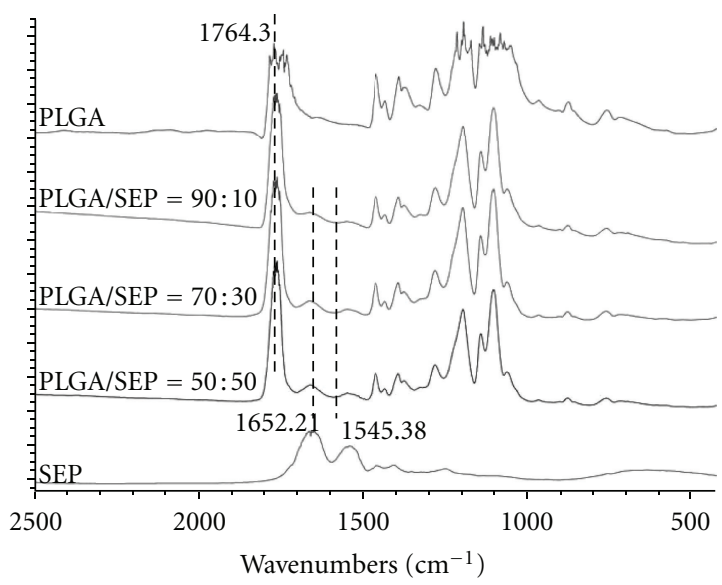

FIgUre 2: FTIR spectra of SEP, pristine PLGA, and SEP/PLGA nanofibers with different blending ratios.

is found to be more favorable, these two methods can also be used to improve the mechanical strength of electrospun fibers. These electrospun SEP/PLGA mats were quite soft and a little stretchy, and much tougher and stronger than the pure SEP film acquired in the previous study. This will also facilitate the ease of trimming, handling, and insertion of the membrane around the affected tooth root and bone. Briefly, blend-electrospinning with PLGA greatly improved the mechanical strength of SEP, which was very fragile and almost impossible to handle.

3.4. FTIR Measurement. FTIR spectra of pristine PLGA electrospun nanofibers, SEP powders, and SEP/PLGA blending electrospun nanofibers with different mixing ratios $(90: 10$, $70: 30$, and $50: 50$ ) are presented in Figure 2. There was a characteristic absorptive band at $1764.30 \mathrm{~cm}^{-1}$ due to the ester groups in the pristine PLGA fibers. For the SEP powders, there were two characteristic amide I and amide II absorptive bands at $1652.21 \mathrm{~cm}^{-1}$ and $1545.38 \mathrm{~cm}^{-1}$ in the spectrum. All these three absorptive bands were observed in the spectra of blending nanofibers with various mixing ratios, and this confirmed the existence of PLGA and SEP in the blending nanofibers. The results also showed that the intensities of amide I and amide II bands were increased with the increase of SEP content in the blended fibers.

3.5. Cell Morphology and Proliferation. Figure 3 shows the SEM photographs of cellular attachment and proliferation on PLGA and SEP/PLGA nanofibers 3 days after the seeding of cells. The morphology of cells growing on the nanofibers was typical for these L929 spindle-shaped fibroblast cells. The results showed that all the electrospun nanofibers could support the attachment, migration, and proliferation of L929 cells which involved the growth, spreading, and wrapping up of pseudopodia. Based on the SEM examinations, cells showed a better spreading and proliferation to confluence on the SEP/PLGA nanofibers compared to those on the pristine PLGA fibers at the same time-point as 3 days after cell seeding. In some areas, the cells almost formed a monolayer by wrapping up around the adjacent nanofibers. In the SEP/PLGA groups, a great number of fine pseudopodia were observed on the fibers with a smaller diameter. Some cells were also observed to migrate into the matrix in all the groups, not just spread on top of the substrate surface which showed the active interaction between the cells and the nanofibers. While in the study, the lower porosity and denser morphology were chosen to fulfill the basic barrier function of the nanofibrous mats considering the future application for GTR membrane.

MTT method was used, and the results are shown in Figure 4 in order to evaluate the in vitro proliferation of L929 cells on the pristine PLGA and SEP/PLGA nanofibers. The results showed that the Formazan absorbance data always increased with the increase in culture time $(P<$ 0.05 ) and this suggests that L929 cells could proliferate on all the electrospun nanofibers. After 1 day, there was no significant difference among all the groups $(P>0.05)$. After 3,5 , and 7 days, the cell viability of SEP/PLGA blending nanofibers $(70: 30$, and $50: 50$ ), was significantly higher than those of pristine PLGA nanofibers and 90:10 SEP/PLGA group $(P<0.05)$. The results in the study indicated that the electrospun SEP/PLGA nanofibers could enhance the attachment, migration, and proliferation of fibroblast cells. These results warrant further investigations into the use of electrospun SEP/PLGA nanofibers for the periodontal GTR application.

\section{Conclusion}

In this study, SEP/PLGA nanofibrous membranes were successfully prepared by blend-electrospinning of SEP and PLGA. These hydrophilic nanofibrous mats had uniform, bead-free and interwoven morphology, which is similar to the ECM and natural eggshell membrane. The mechanical performance of pure SEP has also been greatly improved with the addition of PLGA, which is a major concern for GTR membrane application. In vitro cellular tests showed that the SEP/PLGA nanofibers can positively enhance the proliferation of the cells, as well as promote the cell-matrix interaction. In the pilot study, several biocompatibility tests, such as hemolysis test, oral mucous membrane irritation test, cytotoxicity test, and acute toxicity test, have been done and the results also showed that SEP has very good biocompatibility. However, since the immunogenicity of animal-derived biomaterial is always an important concern for medical use, the next step of our research will be focused on the possible immunogenic response of SEP products. Moreover, designing a novel layered structure, which involves 


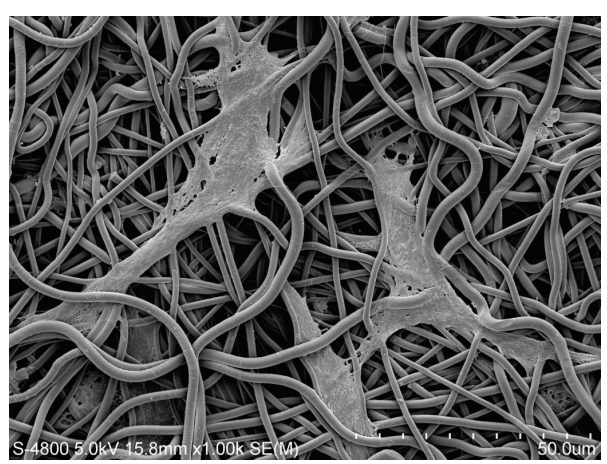

(a)

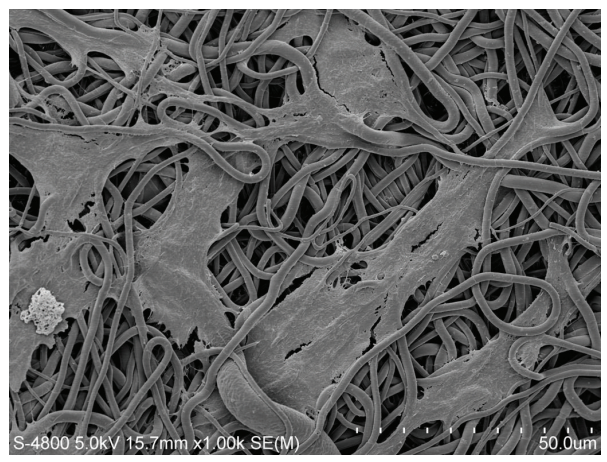

(c)

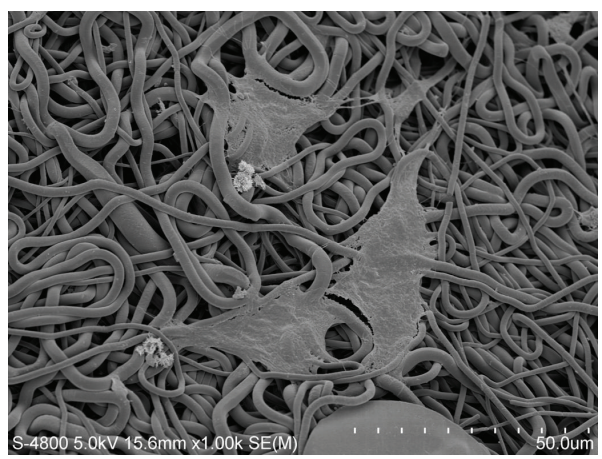

(b)

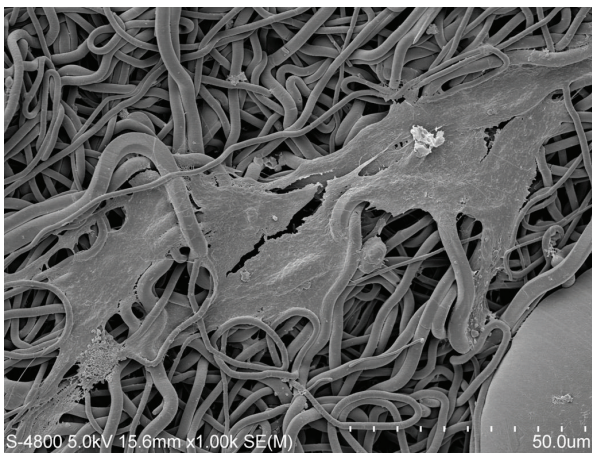

(d)

FIGURE 3: SEM photographs of cellular growth on different groups of nanofibers. (a) pristine PLGA; (b) SEP/PLGA = 90: 10; (c) SEP/PLGA $=70: 30 ;(\mathrm{d})$ SEP/PLGA $=50: 50$.

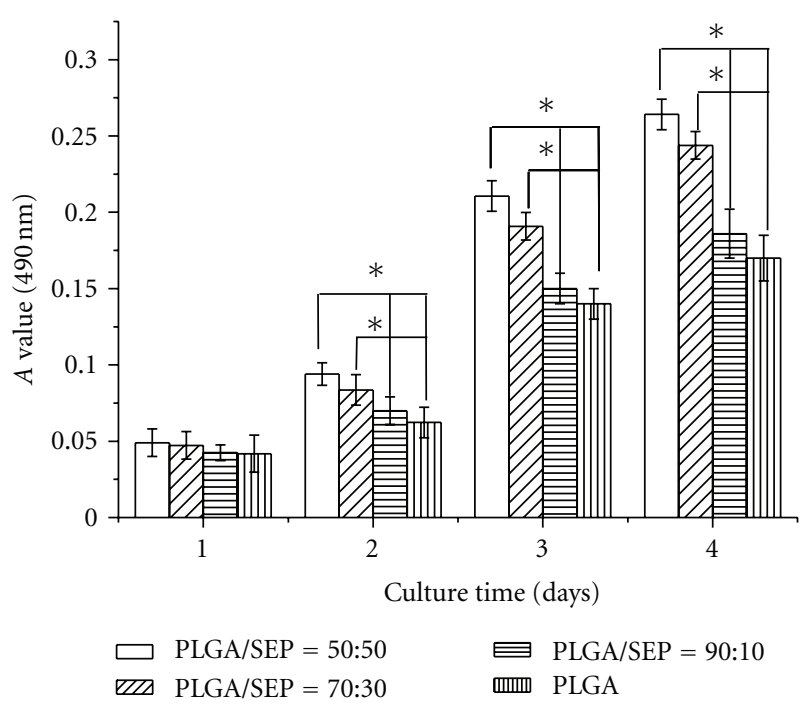

FIGURE 4: Cell proliferation on different groups of nanofibers.

different porosity and composition, will also be included in the following investigations. This will help to simulate the asymmetric structure of natural ESM and meet the different needs of GTR membrane for promoting periodontal tissue and preventing epithelial migration. Although there is still a long way to go to obtain a satisfactory GTR membrane from SEP, this study does indicate the potential of SEP/PLGA nanofibers to be a candidate and also provides a basis for future optimization.

\section{Acknowledgment}

This study is supported by the National Nature Science Foundation of China (Grant no. 30800222).

\section{References}

[1] P. E. Petersen, "The World Oral Health Report 2003: continuous improvement of oral health in the 21st centurythe approach of the WHO Global Oral Health Programme," Community Dentistry and Oral Epidemiology, vol. 31, no. 1, pp. 3-24, 2003.

[2] P. Gentile, V. Chiono, C. Tonda-Turo, A. M. Ferreira, and G. Ciardelli, "Polymeric membranes for guided bone regeneration," Biotechnology Journal, vol. 6, no. 10, pp. 1187-1197, 2011.

[3] A. S. AlGhamdi and S. G. Ciancio, "Guided tissue regeneration membranes for periodontal regeneration-a literature review," Journal of the International Academy of Periodontology, vol. 11, no. 3, pp. 226-231, 2009.

[4] C. M. Cordeiro and M. T. Hincke, "Recent patents on eggshell: shell and membrane applications," Recent Patents on Food, Nutrition \& Agriculture, vol. 3, no. 1, pp. 1-8, 2011.

[5] T. Nakano, N. I. Ikawa, and L. Ozimek, "Chemical composition of chicken eggshell and shell membranes," Poultry Science, vol. 82, no. 3, pp. 510-514, 2003. 
[6] W. T. Tsai, J. M. Yang, C. W. Lai, Y. H. Cheng, C. C. Lin, and C. W. Yeh, "Characterization and adsorption properties of eggshells and eggshell membrane," Bioresource Technology, vol. 97, no. 3, pp. 488-493, 2006.

[7] M. S. Fernandez, K. Passalacqua, J. I. Arias, and J. L. Arias, "Partial biomimetic reconstitution of avian eggshell formation," Journal of Structural Biology, vol. 148, no. 1, pp. $1-10,2004$.

[8] K. Maeda and Y. Sasaki, "An experience of hen-egg membrane as a biological dressing," Burns, vol. 8, no. 5, pp. 313-316, 1982.

[9] Y. Zadik, "Self-treatment of full-thickness traumatic lip laceration with chicken egg shell membrane," Wilderness and Environmental Medicine, vol. 18, no. 3, pp. 230-231, 2007.

[10] J. Y. Yang, S. S. Chuang, W. G. Yang, and P. K. Tsay, "Egg membrane as a new biological dressing in split-thickness skin graft donor sites: a preliminary clinical evaluation," Chang Gung Medical Journal, vol. 26, no. 3, pp. 153-159, 2003.

[11] L. Dupoirieux, D. Pourquier, M. C. Picot, and M. Neves, "Comparative study of three different membranes for guided bone regeneration of rat cranial defects," International Journal of Oral and Maxillofacial Surgery, vol. 30, no. 1, pp. 58-62, 2001.

[12] F. Yi, J. Yu, Z. X. Guo, L. X. Zhang, and Q. Li, "Natural bioactive material: a preparation of soluble eggshell membrane protein," Macromolecular Bioscience, vol. 3, no. 5, pp. 234-237, 2003.

[13] F. Yi, Z. X. Guo, L. X. Zhang, J. Yu, and Q. Li, "Soluble eggshell membrane protein: preparation, characterization and biocompatibility," Biomaterials, vol. 25, no. 19, pp. 4591-4599, 2004.

[14] J. W. Lu, Q. Li, Q. L. Qi, Z. X. Guo, and J. Yu, "Surface engineering of poly(D,L-lactic acid) by entrapment of soluble eggshell membrane protein," Journal of Biomedical Materials Research A, vol. 91, no. 3, pp. 701-707, 2009.

[15] J. Jia, Y. Y. Duan, J. Yu, and J. W. Lu, "Preparation and immobilization of soluble eggshell membrane protein on the electrospun nanofibers to enhance cell adhesion and growth," Journal of Biomedical Materials Research A, vol. 86, no. 2, pp. 364-373, 2008.

[16] I. O. Smith, X. H. Liu, L. A. Smith, and P. X. Ma, "Nanostructured polymer scaffolds for tissue engineering and regenerative medicine," Wiley Interdisciplinary Reviews. Nanomedicine and Nanobiotechnology, vol. 1, no. 2, pp. 226236, 2009.

[17] D. Li and Y. Xia, "Electrospinning of nanofibers: reinventing the wheel?" Advanced Materials, vol. 16, no. 14, pp. 1151-1170, 2004.

[18] T. Courtney, M. S. Sacks, J. Stankus, J. Guan, and W. R. Wagner, "Design and analysis of tissue engineering scaffolds that mimic soft tissue mechanical anisotropy," Biomaterials, vol. 27, no. 19, pp. 3631-3638, 2006.

[19] Q. P. Pham, U. Sharma, and A. G. Mikos, "Electrospinning of polymeric nanofibers for tissue engineering applications: a review," Tissue Engineering, vol. 12, no. 5, pp. 1197-1211, 2006.

[20] K. Ramachandran and P. I. Gouma, "Electrospinning for bone tissue engineering," Recent Patents on Nanotechnology, vol. 2, no. 1, pp. 1-7, 2008.

[21] F. Yi, Z. X. Guo, P. Hu, Z. X. Fang, J. Yu, and Q. Li, "Mimetics of eggshell membrane protein fibers by electrospinning," Macromolecular Rapid Communications, vol. 25, no. 10, pp. 1038-1043, 2004.

[22] J. Kang, M. Kotaki, S. Okubayashi, and S. Sukigara, "Fabrication of electrospun eggshell membrane nanofibers by treatment with catechin," Journal of Applied Polymer Science, vol. 117, no. 4, pp. 2042-2049, 2010.

[23] W. J. Li, C. T. Laurencin, E. J. Caterson, R. S. Tuan, and F. K. Ko, "Electrospun nanofibrous structure: a novel scaffold for tissue engineering," Journal of Biomedical Materials Research, vol. 60, no. 4, pp. 613-621, 2002.

[24] Z. X. Meng, X. X. Xu, W. Zheng et al., "Preparation and characterization of electrospun PLGA/gelatin nanofibers as a potential drug delivery system," Colloids and Surfaces B, vol. 84, no. 1, pp. 97-102, 2011.

[25] G. Wang, X. Hu, W. Lin, C. Dong, and H. Wu, "Electrospun PLGA-silk fibroin-collagen nanofibrous scaffolds for nerve tissue engineering," In Vitro Cellular and Developmental Biology-Animal, vol. 47, no. 3, pp. 234-240, 2011.

[26] D. Xie, H. Huang, K. Blackwood, and S. MacNeil, "A novel route for the production of chitosan/poly(lactide-coglycolide) graft copolymers for electrospinning," Biomedical Materials, vol. 5, no. 6, Article ID 065016, 2010.

[27] J. Stitzel, J. Liu, S. J. Lee et al., "Controlled fabrication of a biological vascular substitute," Biomaterials, vol. 27, no. 7, pp. 1088-1094, 2006.

[28] H. J. Jin, J. Chen, V. Karageorgiou, G. H. Altman, and D. L. Kaplan, "Human bone marrow stromal cell responses on electrospun silk fibroin mats," Biomaterials, vol. 25, no. 6, pp. 1039-1047, 2004.

[29] W. J. Li, R. Tuli, C. Okafor et al., "A three-dimensional nanofibrous scaffold for cartilage tissue engineering using human mesenchymal stem cells," Biomaterials, vol. 26, no. 6, pp. 599-609, 2005.

[30] J. H. He, Y. Q. Wan, and J. Y. Yu, "Effect of concentration on electrospun polyacrylonitrile (PAN) nanofibers," Fibers and Polymers, vol. 9, no. 2, pp. 140-142, 2008.

[31] B. Gupta, C. Plummer, I. Bisson, P. Frey, and J. Hilborn, "Plasma-induced graft polymerization of acrylic acid onto poly(ethylene terephthalate) films: characterization and human smooth muscle cell growth on grafted films," Biomaterials, vol. 23, no. 3, pp. 863-871, 2002.

[32] S. R. Bhattarai, N. Bhattarai, P. Viswanathamurthi, H. K. Yi, P. H. Hwang, and H. Y. Kim, "Hydrophilic nanofibrous structure of polylactide; fabrication and cell affinity," Journal of Biomedical Materials Research A, vol. 78, no. 2, pp. 247-257, 2006.

[33] T. Kawase, K. Yamanaka, Y. Suda et al., "Collagen-coated poly(L-lactide-co- $\varepsilon$-caprolactone) film: a promising scaffold for cultured periosteal sheets," Journal of Periodontology, vol. 81, no. 11, pp. 1653-1662, 2010.

[34] T. Fukushima, T. Hayakawa, Y. Inoue, K. Miyazaki, and Y. Okahata, "Intercalation behavior and tensile strength of DNAlipid films for the dental application," Biomaterials, vol. 25, no. 24, pp. 5491-5497, 2004.

[35] X. Li, W. Lou, and R. Song, "Experimental investigation of Polyurethane electrospun nanofibers mat for nanobiomedical device-relationship between mechanical property and thickness," in the 3rd IEEE International Conference on Nano/Molecular Medicine and Engineering (NANOMED '09), October 2009. 

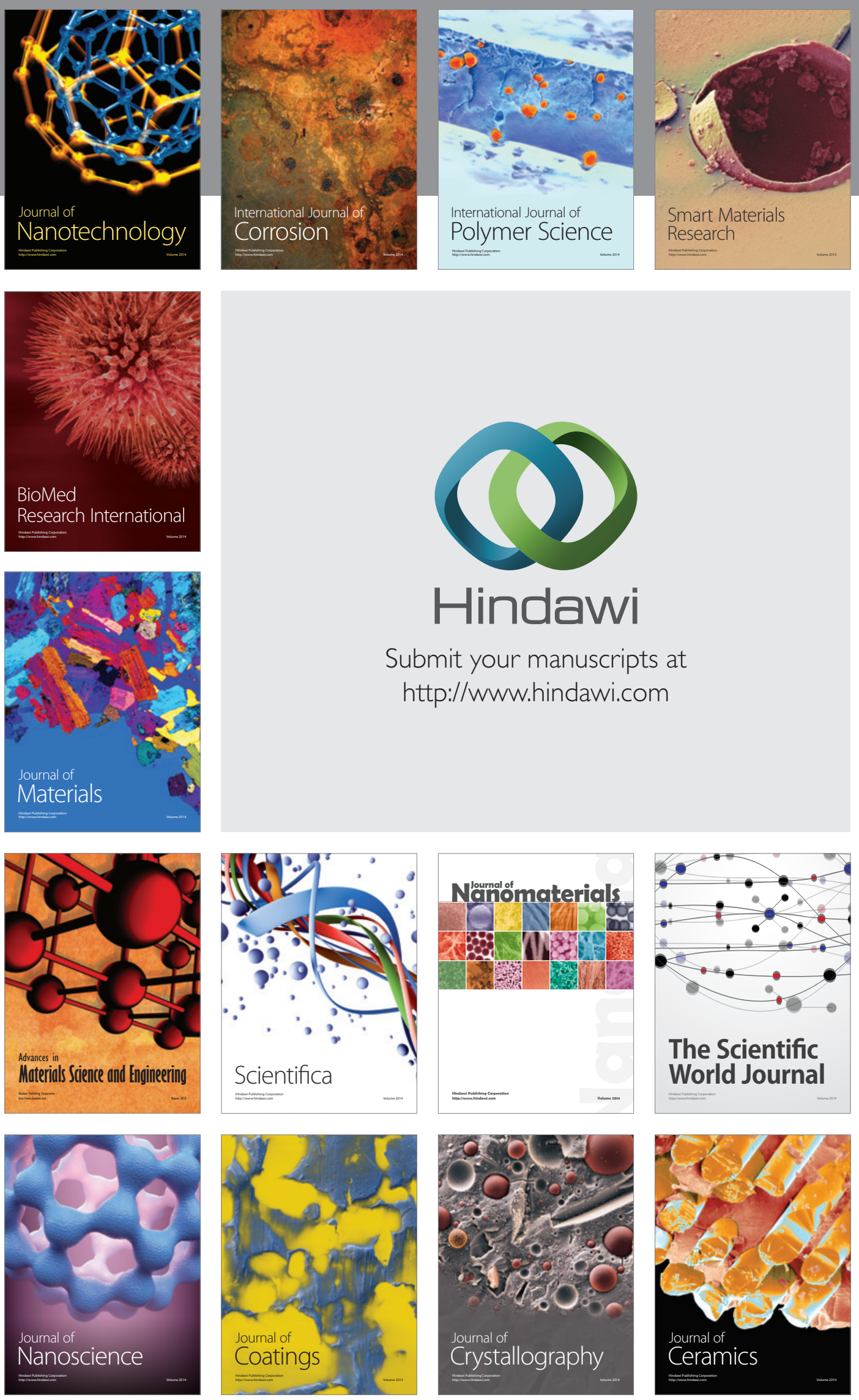

The Scientific World Journal

Submit your manuscripts at

http://www.hindawi.com

\section{World Journal}

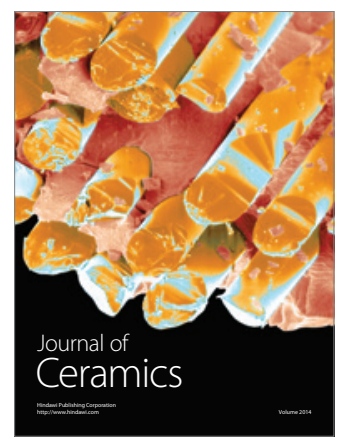

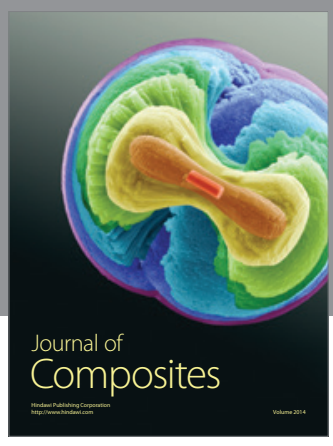
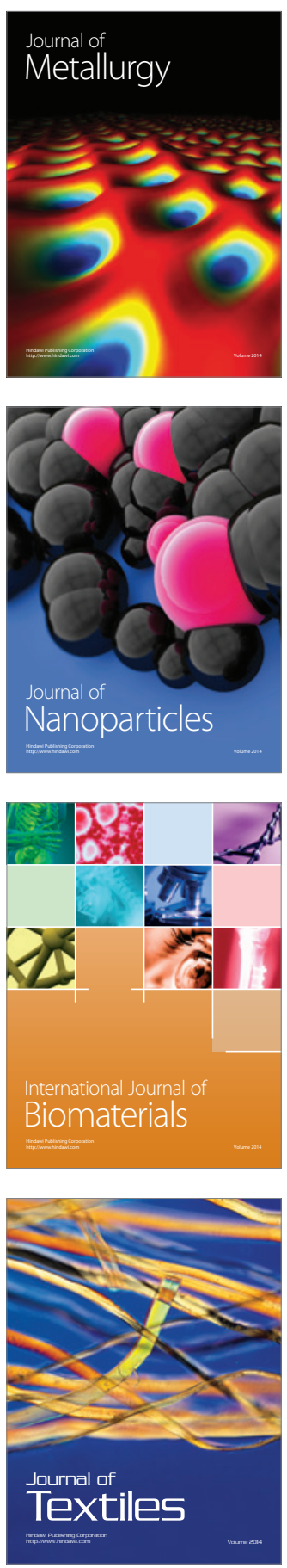